\title{
LAS COMPETENCIAS DEL ESTADO Y DE LAS COMUNIDADES AUTONOMAS ANTE LA LIBRE CIRCULACION DE PRODUCTOS EN EL MERCADO UNICO: EL PROBLEMA DE LA HOMOLOGACION
}

\author{
POR \\ Alicia Camacho Garcia
}

SUMARIO: I. LA ELIMINACION DE FRONTERAS TÉCNICAS EN EL LIBRO BLANCO de Mercado Unico. II. Las directivas del "Nuevo Enfooue". III. La conTROVERSIA COMPETENCIAL EN TORNO A LA HOMOLOGACION TRADICIONAL. IV. LA SEntencia del Tribunal Constitucional 252/88. V. Confluencia de titulo COMPETENCIALES en el "NueVo Enfoque" comunitario de la homologacion DE COMPETENCIAS. VI. ESQUEMA POSIBLE DE ARTICULACION DE COMPETENCIAS.

\section{LA ELIMINACION DE FRONTERAS TECNICAS EN EL LIBRO BLANCO DEL MERCADO UNICO}

En junio de 1985, el Libro Blanco de la Comisión CEE para la consecución del mercado interior levanta acta de las insuficiencias que presenta la armonización prevista en el artículo 100 del Tratado CEE para la libre circulación de productos y la realización del mercado común (1).

Aunque se estima que el método de armonización de disposiciones legales, reglamentarias y administrativas de los Estados miembros mediante la adopción de Directivas que incorporan especificaciones técnicas detalladas ha constituido la piedra angular de la acción de la Comunidad durante sus primeros 25 años, el Libro Blanco pone de relieve también sus defectos: una estrategia totalmente centrada en la armonización da lugar a proliferación normativa, es demasiado lenta y rígida, y podría obstaculizar la innovación.

Se constata la equivalencia esencial de las distintas legislaciones nacionales en cuanto a los objetivos de protección de la salud, la seguridad y el medio ambiente, objetivos comunes que sin embargo se concretan en diferencias en las especificaciones exigidas en las reglamentaciones técnicas nacionales. Como tales diferencias provocan

(1) El texto de este epígrafe es, en extracto, transcripción prácticamente literal del propio Libro Blanco, párrafos 57-78, dedicados a la eliminación de fronteras técnicas en el mercado único. No obstante, se ha modificado en algunos casos el orden de exposición, y sintetizado aclarado algún aspecto. 
obstáculos en los intercambios, la Comisión propone una nueva estrategia que permita progresos más rápidos hacia la eliminación de las fronteras técnicas, combinando el principio del reconocimiento mutuo y el método de la armonización.

La estrategia propuesta se basa en los siguientes principios:

* En las iniciativas que se adopten de cara al mercado interior, debe hacerse una clara distinción entre los aspectos que es fundamental armonizar y aquéllos que pueden ser objeto de reconocimiento mutuo de las normas nacionales.

* La armonización de las legislaciones (Directivas del Consejo basadas en el artículo 100 TCEE) se limitará en lo sucesivo a establecer, de modo obligatorio para todos los Estados miembros, las exigencias esenciales en materia de salud y seguridad, con objeto de permitir la libre circulación de los productos que se ajusten a ellas.

* Se favorecerá al máximo la armonización de las normas industriales mediante la elaboración de normas europeas, pero la falta de normas de este tipo no debe ser un obstáculo para la libre circulación. Mientras se ponen a punto las normas europeas, el principio rector debe estar constituido por la mutua aceptación de las normas nacionales mediante procedimientos acordados.

El Libro Blanco organiza esta estrategia en torno a tres cuestiones: el nuevo método de armonización, la prevención de nuevos obstáculos y el principio de reconocimiento mutuo.

\section{El nuevo método de armonización}

En los sectores en los que los obstáculos a los intercambios procedan de normas nacionales divergentes justificadas en materia de salud y seguridad de los ciudadanos y de protección de los consumidores y del medio ambiente, la armonización de las legislaciones se limitará a establecer las exigencias esenciales a las que deben ajustarse los productos para circular libremente por la Comunidad.

Las especificaciones técnicas detalladas correspondientes se definirán en las normas europeas elaboradas por el Comité Europeo de Normalización (CEN) o por organismos sectoriales de normalización.

\section{Prevención de nuevos obstáculos}

Se extiende el campo de aplicación de la Directiva 83/189/CEE, que establece un procedimiento de información previa a la Comisión CEE de los proyectos de normas técnicas de los Estados miembros, procedi- 
miento que desde su adopción para determinados productos industriales se ha revelado eficaz para prevenir obstáculos a la libre circulación.

\section{Reconocimiento mutuo}

De acuerdo con la jurisprudencia del Tribunal de Justicia, en el caso de que la armonización de las normas no se considere esencial desde el punto de vista de la salud y seguridad o desde el punto de vista industrial, ha de aplicarse el principio del reconocimiento mutuo, en virtud del cual los bienes fabricados y comercializados legalmente en un Estado miembro deben tener libre acceso a los demás Estados miembros.

Aplicando el principio del reconocimiento pleno y total de las diferentes normas de calidad, no se podrá, en particular, prohibir la venta de determinados productos únicamente porque hayan sido fabricados con arreglo a especificaciones diferentes de las aplicadas en el Estado importador.

El comprador no tiene por qué demostrar la equivalencia de un producto fabricado con arreglo a las normas vigentes en el Estado exportador. Tampoco puede obligársele a someter a dicho producto a pruebas técnicas suplementarias ni a procedimientos de certificación en el Estado importador.

Para evitar repeticiones inútiles de determinados procedimientos de prueba y certificación, se prevé el establecimiento de condiciones y códigos de práctica comunes que vinculen a los laboratorios y organismos competentes en materia de certificación.

\section{LAS DIRECTIVAS DEL “NUEVO ENFOQUE"}

A partir del planteamiento expuesto, la Comunidad Europea comienza a adoptar Directivas que, recogiendo este "nuevo enfoque", suponen un giro sustancial en relación con las actuaciones que las Administraciones de los Estados miembros han de realizar para controlar la comercialización de productos fabricados en su propio territorio o en el de los restantes Estados miembros.

Los rasgos principales de esta Directivas (2) son los siguientes:

(2) Se han consultado para este resumen la Directiva $88 / 378 / \mathrm{CEE}$. de 3 de mayo, relativa a la aproximación de las legislaciones de los Estados miembros sobre la seguridad de los juguetes, y la Directiva 89/686/CEE, de 21 de diciembre, sobre aproximación de las legislaciones de los Estados miembros relativas a los equipos de protección individual. 
* Se regulan únicamente la exigencias esenciales (de salud, seguridad, etc.) que deben cumplir los productos comercializados, sin establecer especificaciones técnicas detalladas, que quedan en manos de los organismos europeos de normalización.

* Tales exigencias se establecen, con carácter indistinto, para la puesta en mercado del producto en cualquier punto del territorio comunitario; se trata de la llamada "armonización total", que no admite ya regulaciones diferentes para los productos que sólo se comercialicen en el territorio del Estado miembro en que han sido fabricados.

* Se instituye la "marca CE", que acredita que un producto cumple las exigencias esenciales de la Directiva. La "marca $\mathrm{CE}^{\prime \prime}$, que permite que un producto pueda circular sin traba alguna por todo el territorio comunitario, puede obtenerse mediante distintos procedimientos:

- Puede estamparla el fabricante bajo su propia responsabilidad, en caso de que el producto cumpla la normativa técnica adoptada por los organismos europeos de normalización, o bien, en ciertos casos en que, tratándose de productos de diseño sencillo y bajo riesgo, el fabricante se hace directamente responsable de la conformidad del producto con las exigencias esenciales.

En ambos supuestos, el fabricante debe efectuar una "declaración de conformidad", que quedará a disposición de las autoridades competentes, antes de estampar la "marca CE" en el producto.

- Los restantes productos deben someterse a los correspondientes ensayos y verificaciones técnicas, llevados a cabo por un organismo público o privado acreditado por la Administración, que (en caso de resultar favorables) emite un "certificado CE de tipo", que acredita que el producto cumple las exigencias esenciales.

Provisto del "certificado CE de tipo" expedido por un organismo acreditado, el proceso es idéntico al anterior: el fabricante efectúa la "declaración de conformidad" y estampa la "marca CE".

* Se regulan asimismo: los mecanismos de control de la producción (encomendados normalmente a los organismos acreditados); los controles del producto puesto en el mercado, que pueden implicar la retirada de productos conformes con las exigencias esenciales cuando se compruebe que los mismos pueden (a pesar de dicha conformidad) afectar a la salud o seguridad; $y$ las medidas relativas a aquellos productos que, no sien- 
do conformes con dichas exigencias esenciales, lleven estampada la "marca CE".

* Se establecen las condiciones que deben reunir los organismos para poder ser acreditados por la Administración para el cumplimiento de las funciones que se les encomiendan, así como la posibilidad de retirada de la acreditación cuando el organismo deje de cumplir los requisitos establecidos.

Este es el esquema de actuaciones que, en el horizonte del mercado único, sustituirá progresivamente a la tradicional actividad administrativa de homologación y normalización, y que sin duda tiene un importante impacto en el actual panorama competencial en relación con estas actuaciones administrativas, fuertemente conflictivas ya antes de la aparición del "nuevo enfoque".

\section{LA CONTROVERSIA COMPETENCIAL EN TORNO A LA HOMOLOGACION TRADICIONAL}

Sintéticamente, las fases del proceso tradicional de normalización y homologación de productos son las siguientes (3):

* Determinación de los productos sometidos a normalización por razones de seguridad industrial.

* Aprobación de la normativa técnica correspondiente.

* Homologación de prototipos, tipos y modelos: esta actividad de carácter ejecutivo implica el reconocimiento oficial del cumplimiento por aquéllos de lo establecido en un reglamento, norma o instrucción técnica obligatoria.

* Certificados de conformidad de la producción: procedimiento de control de la producción en serie, con la finalidad de comprobar periódicamente el cumplimiento de las condiciones que sirvieron de base a la homologación.

* Acreditación de Laboratorios de ensayo y Entidades de control e inspección reglamentarias (ENICRES): entidades privadas que colaboran con la Administración en materia de seguridad industrial, bien mediante la realización de pruebas o ensayos exigidos con motivo de la homologación de un prototipo, bien en ac-

(3) La normativa reguladora se contiene principalmente en el Reglamento General de las actuaciones del Ministerio de Industria y Energia en el campo de la normalización y homologación, aprobado por Real Decreto 2584/81, de 18 de septiembre, y modificado por los Reales Decretos 734/85, de 20 de febrero, y 105/88, de 12 de febrero; y en el Real Decreto 1407/87, de 13 de noviembre, que regula las Entidades de inspección y control reglamentario en materia de seguridad de los productos, equipos e instalaciones industriales. 
tividades conexas (revisiones, inspecciones, auditorias de empresas, etc.).

No ha sido cuestionada la competencia estatal en la esfera normativa (que abarcaría las dos primeras fases señaladas), por lo que no parece necesario extenderse sobre esta cuestión. Baste únicamente señalar que la reserva a favor del Estado de la "normativa sobre seguridad industrial" que aparece en los Estatutos de Autonomía explica que la normativa estatal en este campo haya sido pacíficamente admitida por las Comunidades Autónomas, salvo en lo que afecta a la atribución de funciones ejecutivas.

En efecto, la retención estatal de la función homologadora y conexas ha sido fuente constante de conflictividad con las Comunidades Autónomas vasca y catalana (son once los conflictos pendientes actualmente de pronunciamiento del Tribunal Constitucional, interpuesto por dichas Comunidades contra normas o resoluciones estatales).

Los argumentos esgrimidos por el Estado para defender su competencia (con variantes debidas a los distintos fines perseguidos por la homologación en cada caso protección de la salud y seguridad, protección del medio ambiente, defensa de los consumidores, etc.-) podrían esquemáticamente resumirse así:

a) La homologación constituye una condición de lícito acceso del producto al mercado, lo que permite amparar la competencia estatal en la ordenación económica general (artículo 149.1.13a CE).

b) La igualdad de condiciones básicas en la protección del consumidor (artículo 149.1.1a en relación con el artículo 51 CE).

c) La prevalencia de títulos más específicos en función de los objetos perseguidos por la homologación, que configurarian la competencia estatal como una "función ejecutiva básica" basada en razones de protección de la Salud (artículo 149.1.16 $\mathrm{CE}$ ); protección del medio ambiente (artículo 149.1.23 $\mathrm{CE}$ ), seguridad (artículo 149.1.29a CE), etc.

d) El título relativo al comercio exterior (artículo 149.1.10 $\mathrm{CE}$ ) para los productos importados.

e) El principio de territorialidad de la competencia autonómica, que excluiría la eficacia nacional de las resoluciones de homologación de las Administraciones autonómicas.

Todo ello, contrapuesto a la tesis principal de las Comunidades Autónomas, según la cual la homologación es un acto administrativo que forma parte de la función ejecutiva en materia de industria, base de su reivindicación competencial. 
Para completar el esbozo de esta polémica cuestión, deben reseñarse aún dos datos importantes:

- Toda la conflictividad existente en la actualidad se refiere a normas o actos estatales impugnados por las Comunidades Autónomas; aunque no sin debate, el Estado no ha impugnado ante el Tribunal Constitucional las normas dictadas por las Comunidades Autónomas vasca y catalana reguladoras de sus actividades en el campo de la normalización y homologación (4), ni las resoluciones de homologación dictadas por las mismas en aplicación de dicha normativa (con excepción de las relativas a productos importados).

- Las restantes Comunidades Autónomas no han reivindicado ante el Tribunal Constitucional las funciones de homologación y conexas, a pesar de que sus Estatutos de Autonomía les atribuyen competencia ejecutiva en materia de industria (con la excepción de Cantabria). Ello pudiera deberse a la expresa reserva a favor del Estado contenida en los Reales Decretos de traspasos, aunque no se necesario incidir aquí sobre la debilidad de este argumento en el caso hipotético de que se planteara un conflicto de competencias.

En este estado de cosas, se vislumbra un acercamiento de posturas, al menos parcial, a finales del pasado año: por cuerdo de 21.12.89, el Consejo de Ministros acepta un requerimiento de incompetencia formulado por el Gobierno Vasco en relación con una Resolución de homologación de la Dirección General de Industria. La aceptación del requerimiento vasco implica el reconocimiento de la competencia de la Comunidad Autónoma para homologar los productos fabricados en su territorio, por tratarse la función homologadora de una actividad de naturaleza ejecutiva en materia de industria.

No parece exagerado afirmar que el giro en la posición estatal (influido, sin duda, por el pronunciamiento del Tribunal Constitucional en la Sentencia $52 / 88$ sobre la Ley catalana del Juego) podría afectar a algunos conflictos pendientes, aunque la operatividad de otros títulos competenciales llevaría posiblemente a soluciones distintas en un análisis caso por caso.

El propósito de este trabajo, sin embargo, no es este análisis, sino conectar la distribución interna de competencias con el "nuevo enfoque" comunitario sobre la homologación, para intentar responder a

(4) Cataluña: Orden de 5 de marzo de 1986 (D.O. 12.3.86), de asignación de funciones en el campo de la homologación y la aprobación de prototipos, tipos y modelos, modificada por la Orden de 30 de mayo de 1986 (D.O. 6.6.86), a raiz de un requerimiento de incompetencia. Pais Vasco:Decreto 275/1986, de 25 de noviembre, sobre calidad y seguridad industrial (B.0.13.12.86) 
una pregunta: ¿cómo afectan las nuevas Directivas comunitarias a la competencia autonómica para la homologación de productos industriales de fabricación nacional, basada en su habilitación estatutaria sobre la industria? 0 , dicho de otro modo, las actuaciones administrativas que las Directivas prevén en este campo, ¿han de considerarse, sin mayores matizaciones, encuadradas en el título estatal exclusivo sobre el comercio exterior, al tratarse de actos que dan a la producción nacional acceso directo a todo el territorio comunitario?.

\section{SENTENCIA DEL TRIBUNAL CONSTITUCIONAL 252/88}

Aunque la Sentencia enjuicia un conflicto de características distintas a la cuestión aquí analizada parece oportuno traer a colación las reflexiones del Tribunal Constitucional sobre las consecuencias del proceso de incorporación de España a la CEE.

La Sentencia sienta, en primer lugar, doctrina sobre la neutralidad de la integración europea en el orden interno de distribución de competencias: "Son, en consecuencia, las reglas internas de distribución competencial las que en todo caso han de fundamentar la respuesta a los conflictos de competencia planteándose entre el Estado y las Comunidades Autónomas, las cuales, por la misma razón, tampoco podrán considerar ampliado su propio ámbito competencial en virtud de una conexión internacional". (F.J.2).

Pero también proporciona ciertas pautas que completan ese punto de vista, no solo para el concreto conflicto analizado, sino también extrapolables a otras actuaciones administrativas afectadas por el proceso de integración:

" (...) tanto la necesidad de proporcionar al Gobierno los instrumentos indispensables para desempeñar la función que le atribuye el artículo 93 CE (...) como, de otra parte, el hecho de que sea la Administración Central del Estado (...) la competente para realizar actuaciones que, cuando no está presente esa conexión con el exterior, corresponden a la Administración Autonómica, obligan a articular el ejercicio de las competencias propias del Estado y de la Comunidad Autónoma de modo tal que uno y otra, sin invadir el ámbito competencial ajeno, no obstaculicen el desempeño de las funciones que la Constitución y los Estatutos le atribuyen ni echen cargas innecesarias sobre los administrados. (...) Tanto de la interpretación sistemática de la Constitución como de la supremacia de ésta sobre los Estatutos se deriva la necesidad de colaboración entre la Administración Central y las Administraciones Autonómicas que puede exigir en muchos casos, sobre todo, con relación a nuestra incorporación a la CEE, formas de articulación (por ejemplo, realiza- 
ción por la Administración Autonómica de tareas de competencia estatal, con sumisión, en consecuencia, y sólo en cuanto a ellas, a instrucciones y supervisión de la Administración central) que sólo una interpretación inadecuada de los preceptos constitucionales y estatutarios puede obstaculizar" (F.J.2).

$Y$ nótese que la constante llamada de la Sentencia (en éste y otros pasajes) al principio de colaboración entre el Estado y la Comunidad Autónoma competente ratione loci seda en un supuesto que sólo afecta al comercio intracomunitario, a una "regulación específica para los intercambios comerciales exteriores, en el marco de la CEE, regulación aún hoy separada de la que ordena el comercio interior ..." (F.J. 3) ¿No habria que proyectar, con mayor intensidad si cabe, esta exigencia de articulación y colaboración en el ejercicio de las competencias en regulaciones que ya no son especificas para los intercambios entre Estados miembros, sino que regulan de modo uniforme la comercialización de productos en el seno del mercado único, sin consideración al destino de los productos?.

Parece que la respuesta ha de ser necesariamente afirmativa, si se admite que, en el conjunto de actuaciones que prevén las Directivas del "nuevo enfoque", confluyen la habilitación autonómica para la ejecución en materia de industria (u otras posibles materias afectadas) y la habilitación estatal para la ejecución en materia de comercio exterior.

\section{CONFLUENCIA DE TITULOS COMPETENCIALES EN EL "NUEVO ENFOQUE" COMUNITARIO DE LA HOMOLOGACION DE PRODUCTOS}

La incidencia de la competencia exclusiva estatal sobre el comercio exterior parece clara, si se tiene en cuenta que la obtención de la "marca CE" da acceso a un producto a su comercialización, sin otros trámites, en todo el territorio comunitario.

Esta conexión exterior se confirma en una lectura detallada de las Directivas, de las que podría destacarse (aparte del papel del Estado como interlocutor ante la CEE y con los restantes Estados miembros en las múltiples comunicaciones y actuaciones coordinadas que se regulan) el hecho de que, en principio, nada parece impedir que un fabricante español acuda a un organismo acreditado en otro Estado miembro para la obtención del "certificado CE de tipo", o viceversa.

En lo que se refiere a las Comunidades Autónomas, la competencia ejecutiva que los Estatutos les atribuyen en materia de industria, como se fundamenta en el citado Acuerdo del Con- 
sejo de Ministros lo en otras materias que, como luego se verá, pudieran resultar prevalentes, atendiendo a los objetivos de la homologación), llevaría a atribuir a éstas las actuaciones relativas a la producción fabricada o comercializada en su territorio. Parece claro que la sustitución de la homologación como acto administrativo por las funciones propias del "nuevo enfoque", de acreditación de organismos y control del mercado, en otras palabras, la modificación del modus operandi de la Administración, no altera en sí misma el encuadramiento material de la competencia. Ambos modos de actuación pertenecerían al ámbito de actuaciones de naturaleza ejecutiva dentro de la misma materia de industria, de competencia autonómica, que si bien puede quedar modulada, no es desplazada por la señalada conexión exterior de la actividad (5).

Y ello sin descartar que, en atención al contenido y finalidades de las Directivas, puedan resultar prevalentes otros títulos especificos sobre los cuales concurra asimismo la competencia ejecutiva autonómica:

- La Directiva 89/686/CEE, relativa a los equipos de protección individual, bien podría entenderse como equivalente a lo que en nuestro ordenamiento interno es la normativa sobre seguridad e higiene en el trabajo (que regula la homologación de estos equipos) que forma parte de la legislación laboral, lo que quizá llevaría a considerar, en este caso, la distribución de competencias para su ejecución, con carácter prevalente a los títulos estatutarios relativos a la industria.

- Tampoco resulta difícil conectar los objetivos de la Directiva $88 / 378 / C E E$, relativa a la seguridad de los juguetes, con la protección de la salud de los consumidores contra los riesgos derivados de tales productos, conectando su transposición, en consecuencia, con la materia sanitaria (6).

Del mismo modo, otros productos acogidos o que se acojan en esta nueva estrategia comunitaria podrian verse desde distinta perspectiva competencial (v. gr. telecomunicaciones, automóviles, sector eléctrico, etc.).

(5) Conflictos positivos de competencia no s. 598/1986, 1403/1986 y 857/1988, interpuestos por la Generalidad de Cataluña contra escrito de 26 de febrero de 1986 del Director General de Salud Pública del Ministerio de Sanidad y Consumo al Director General de Sanidad y Salud Pública del Departamento de Sanidad y Seguridad Social de la Generalidad, y contra los Reales Decretos 1754/1986, 1755/1986 y 1728/1987, sobre intercambio intercomunitario de carnes.

(6) La Directiva 88/378/CEE ha sido objeto de transposición mediante Real Decreto 880/1990, de 29 de junio, publicado en el BOE de 12 de julio. 


\section{ESQUEMA POSIBLE DE ARTICULACION DE COMPETENCIAS}

La articulación de competencias entre el Estado y las Comunidades Autónomas en la línea señalada por la Sentencia 252/88 deviene asi una exigencia tanto de orden juridico-constitucional como, no se olvide, de mera racionalidad y economía administrativas (y este aspecto, aunque no sea tratado aquí de modo directo, es en última instancia indisociable del leitmotiv de las estrategias definidas por la Comunidad Europea para la eliminación de obstáculos en el funcionamiento del mercado único, y de lo que el propio Tribunal Constitucional recuerda cuando alude a modos de articulación de las competencias que no echen cargas innecesarias sobre los administrados).

En todo caso, por lo ya dicho, la articulación de competencias que se propone tiene como orientaciones generales las siguientes:

* Al Estado le corresponde establecer los criterios normativos, transponiendo las Directivas y complementándolas en su caso.

* A las Comunidades Autónomas con competencia ejecutiva en las materias que en cada supuesto resulten prevalentes (industria, ejecución de legislación laboral, comercio interior, sanidad interior, etc.), deben corresponderles las funciones de ejecución, esto es, la aplicación de la normativa estatal.

* Estas competencias ejecutivas de las Comunidades Autónomas que, por la confluencia de materias diversas ya apuntada, supone que aquéllas "desplacen" al Estado de funciones ejecutivas que son propias también de éste último, deben tener como correlato los criterios apuntados por el Tribunal Constitucional en la citada Sentencia 252/88: "sumisión, en consecuencia, y sólo en cuanto a ellas, a instrucciones y supervisión de la Administración central".

De esta forma, la confluencia de las habilitaciones impone la colaboración entre las Administraciones estatal y autonómica y determina la articulación de sus competencias respectivas, garantizando a la vez las funciones ejecutivas ordinarias de las Comunidades Autónomas con las competencias asimismo ejecutivas y de responsabilidad internacional del Estado.

A partir de estas premisas, cabria hacer una tentativa de articulación de las competencias del Estado y de las Comunidades Autónomas, en relación con las principales actuaciones administrativas derivadas de las Directivas.

El esquema que a continuación se expone no constituye, desde luego, un modelo cerrado y rígido, sino que se propone como uno entre los posibles modelos a debatir. Su mayor o menor coincidencia con los sistemas que en la práctica se articulen dependerá de multitud de factores,entre ellos, el propio contenido de las Directivas, que 
presentan rasgos comunes, pero no contenidos idénticos; y sin duda, del grado de acuerdo que pueda alcanzarse entre las Administraciones implicadas a la hora de concretar los mecanismos de colaboración que serían el sustrato de todo modelo integrado de ejercicio de las competencias. De ahí que el esquema no tenga otra pretensión que constituir un posible punto de partida para un debate.

Como, por otra parte, no se pretende un análisis exhaustivo de una Directiva concreta, sino proponer a grandes rasgos un esquema de articulación, se utilizará la expresión "Estado" cuando se trate de competencias que corresponden a éste en exclusiva, en virtud de sus habilitaciones sobre el comercio exterior o las relaciones internacionales, y la expresión "Administración competente" para hacer referencia indistinta a las Comunidades Autónomas (cuando hayan asumido competencia ejecutiva en la materia a considerar) o a la Administración del Estado (cuando,debido a la heterogeneidad estatutaria, ésta haya retenido dicha competencia ejecutiva).

\section{a) Acreditación de los organismos encargados de efectuar los procedimientos de certificación}

La constatación de que estos organismos cumplen la normativa vigente correspondería a la Administración competente para la ejecución de la materia que sea prevalente en cada caso (bien entendido que, por lo antes indicado, cuando la materia sea el comercio o la sanidad, la referencia a estos aspectos la constituirán el comercio o sanidad "interiores").

A esta Administración competente correspondería, pues, tramitar el expediente de acreditación, comprobar el cumplimiento de los requisitos exigibles y dictar la resolución que corresponda.

A la Administración competente correspondería asimismo la comprobación periódica de que el organismo continúa reuniendo los requisitos que, en su día, dieron lugar a la acreditación, y proceder a su anulación en caso contrario.

Al Estado le corresponderían dos funciones relevantes:

- De un lado, coordinar mediante instrucciones las funciones de acreditación que realicen las Comunidades Autónomas, supervisando dichas funciones en los casos en que ello resultara necesario (Sentencia 252/88). En todo caso, estas actuaciones estatales deben configurarse de forma restrictiva y, según se expondrá después, en un marco de colaboración intensa.

- De otro, la notificación a la Comisión CEE y a los restantes Estados de las acreditaciones y anulaciones que se hayan producido. 
También sería la Administración competente la encargada de recibir las notificaciones que los organismos acreditados deben efectuar, en relación con la expedición de los "certificados CE de tipo", y su retirada, y con las medidas adoptadas por los organismos acreditados cuando a éste se encomienda el control de la producción y constaten defectos en la misma. De tales notificaciones la Administración competente habria de dar traslado, normalmente, al Estado, por razones de coordinación y cuando asi venga exigido por ser necesaria su comunicación a la Comisión CEE o a los restantes Estados miembros.

\section{b) Revisión administrativa de las actuaciones de los orga- nismos}

De modo genérico, las Directivas prevén la existencia de vías de recurso para cualquier decisión que adopten los Estados miembros en su aplicación y que conduzcan a una restricción en la comercialización de los productos.

Aunque esta cuestión parece más directamente conectada con las medidas administrativas de retirada de los productos del mercado, suscita la cuestión de la posible revisión administrativa, en vía de recurso, de las actuaciones de los organismos acreditados que supongan una restricción en la comercialización de los productos.

En concreto, se trataria de estudiar las vías de recurso del fabricante ante la denegación o retirada por un organismo acreditado de los "certificados CE de tipo". En principio, parece que esta eventual revisión habría de corresponder a la Administración competente para la acreditación.

\section{c) Presentación por los fabricantes de las "declaraciones de conformidad"}

Estas declaraciones deben ser formuladas por los fabricantes (directamente o previa obtención del "certificado CE de tipo" expedido por un organismo acreditado), a fin de poderlas presentar a las autoridades competentes, según las Directivas, lo que puede interpretarse en el sentido de que éstas dejan en libertad a los Estados miembros para establecer:

- Que los fabricantes han de presentar copia de la declaración de conformidad en el momento de iniciar la comercialización del producto.

Este trámite podría efectuarse en colaboración entre ambas Administraciones, de modo que el fabricante presente 
la "declaración de conformidad" ante la Administración competente, que remitirá copia al órgano estatal que recopile de forma centralizada esta información.

- Que los fabricantes deben estar en disposición de presentar la declaración de conformidad cuando sean requeridos para ello con ocasión de una inspección o de otras medidas de control.

Esta posibilidad se tratará en relación con las medidas excepcionales o de inspección que puedan adoptarse según las Directivas.

\section{d) Control de producción}

Si este control recae bajo la responsabilidad de los organismos acreditados, las medidas adoptadas por éstos habrian de notificarse a la Administración competente, según se vio en el apartado a). Si existe la posibilidad de control administrativo directo de la producción, éste corresponderá a la Administración competente, sin perjuicio de las notificaciones que fuera preciso efectuar al estado en caso de anomalías que dieran lugar a la retirada de la "marca CE".

Sería por tanto la Administración competente la que tendría acceso a la documentación técnica que las Directivas disponen deben tener los fabricantes como soporte de la "declaración de conformidad".

\section{e) Medidas de retirada del producto del mercado por razo- nes de seguridad}

Las Directivas establecen que, cuando un Estado miembro compruebe que un producto provisto de la "marca CE" puede presentar riesgos para la salud o la seguridad, adoptará las medidas pertinentes para su retirada del mercado, prohibiendo su comercialización o libre circulación.

Esta decisión implica la inmediata puesta en marcha de un procedimiento de consultas, tras las cuales la Comisión CEE dictamina si las medidas adoptadas están o no justificadas.

En principio, la competencia para adoptar tales medidas ha de atribuirse a la Administración competente, a quien corresponderá apreciar la concurrencia de riesgos para la salud o seguridad que pueden dar lugar a la retirada o inmovilización de los productos.

Este planteamiento, sin embargo, habria de quedar matizado en dos aspectos: 
- Se deberá poner en inmediato conocimiento del Estado la adopción de la medida de retirada o inmovilización del producto, a efectos de su extensión al resto del territorio nacional y de la iniciación del procedimiento comunitario previsto en las Directivas.

- La posibilidad de intervención directa de la Administración del Estado cuando concurra urgente necesidad ha sido admitida por la jurisprudencia constitucional (Sentencias $33 / 82$ y $15 / 89$, si bien con una connotación de excepcionalidad, atendiendo a situaciones de extrema y generalizada gravedad del riesgo.

\section{f) Medidas relativas a productos no conformes que lleven la "marca CE"}

Las Directivas hacen referencia a las "medidas oportunas" que deben adoptar los Estados miembros para estos productos, sin mayores precisiones; su transposición, al concretar estas medidas, podría contemplar uno o varios aspectos:

- Supervisión administrativa de los "certificados CE de tipo" expedidos por los organismos acreditados, que deben conservar el expediente tramitado a disposición de la Administración.

- Posibilidades de inspección de la producción comercializada bajo la exclusiva responsabilidad del fabricante, a que se ha hecho alusión en el apartado d).

- Régimen de infracciones y sanciones derivadas del incumplimiento: responsabilidad de organismos y fabricantes, según los casos.

- Instancias técnicas y administrativas que deben dictaminar que un producto no es conforme, aunque vaya provisto de la "marca CE" y haya cumplido los requisitos formales exigidos.

\section{g) Funciones generales del Estado}

El esquema propuesto no quedaría completo sin aludir a las funciones generales que necesariamente corresponderian al Estado (aparte de las detalladas en los distintos apartados).

Se trataría de concretar en qué consisten las posibles instrucciones y supervisión que la Sentencia 252/88 cita, como ejemplo de posible articulación de competencias, cuando las Comunidades Autónomas realicen tareas de competencia estatal. 
A ello habría que añadir la necesaria coordinación que complejidad de las actuaciones exige.

Pero ¿cuáles son, de las descritas, las tareas de competencia estatal en sentido estricto? ¿donde termina la competencia sobre "industria" para pasar a convertirse en "comercio exterior"?. No parece posible distinguir los distinguir los procedimientos de control de la producción y comercialización a estos efectos competenciales en una fase concreta, sin desvirtuar su propia naturaleza unitaria.

Lo lógico sería concretar y diseñar estas actuaciones de supervisión y coordinación mediante acuerdo entre el Estado y las Comunidades Autónomas, de modo que abarcaran el completo proceso regulado en las Directivas, creando un órgano ad hoc que, con la participación de ambas Administraciones, fuera encargado de velar por el correcto y coordinado desarrollo de las actividades de todos los órganos llamados a intervenir.

Como podrá apreciarse, el esquema propuesto gravita integramente en el ejercicio de competencias en colaboración, renunciando a priori a una distinción nítida de esferas competenciales en función de la conexión exterior o interior de la actividad, porque todo apunta (7) a que, en el seno del mercado único, esta frontera está llamada a desaparecer progresivamente.

Bien podria recordarse aqui, a modo de conclusión, la importancia que el Libro Blanco atribuye al principio de reconocimiento mutuo $u$ el énfasis puesto en evitar repeticiones inútiles de los procedimientos de control. Y si ésta es la estrategia de la Comunidad Europea para favorecer la libre circulación de productos, idénticos principios deberían inspirar, mutatis mutandis, la transposición de las Directivas del "nuevo enfoque".

5 de noviembre de 1990

(7) El D.O.C.E. de 27.6 .90 publica una propuesta de Directiva relativa a la seguridad general de los productos, que se aplicaria en defecto de norma comunitaria más específica, y cuya aprobación supondría la extensión de los principios y actuaciones administrativas propios del "nuevo enfoque" a toda clase de productos manufacturados, elaborados o agrícolas. 
REALA-1991, núm. 250. CAMACHO GARCIA, ALICIA. LAS COMPETENCIAS DEL ESTADO Y DE L...

\section{CRONICAS}


